\title{
Optimal Surrender Strategies for Equity-Indexed Annuity Investors with Partial Information
}

\author{
Jiaqin Wei, Rongming Wang ’and Hailiang Yang ${ }^{\ddagger}$
}

\begin{abstract}
In this paper we consider an equity-indexed annuity (EIA) investor who wants to determine when he should surrender the EIA in order to maximize his logarithmic utility of the wealth at surrender time. We model the the dynamic of the index using a geometric Brownian motion with regime switching. To be more realistic, we consider a finite time horizon and assume that the Markov chain is unobservable. This leads to the optimal stopping problem with partial information. We give a representation of the value function and an integral equation satisfied by the boundary. In the Bayesian case which is a special case of our model, we obtain analytical results for the value function and the boundary.
\end{abstract}

Keywords: Equity-indexed annuity; Regime switching; Optimal stopping; Partial information; Logarithmic utility

\section{Introduction}

An equity-indexed annuity (EIA) is an equity-linked deferred annuity whose returns are based on the performance of a stock index. The sales of EIAs have grown dramatically in recent years. Because of their popularity, EIAs have received considerable attention in the actuarial literature.

There are two main topics in the study of EIAs. One is the valuation of EIAs. There is a vast of literature on this topic, such as Tiong [19], Moore and Young [14], Lin and Tan [11] and Yuen and Yang [20], among others. Since the EIA investors are entitled the right to surrender the product prior to maturity. So it is important for the investors to determine the optimal surrender time. This leads to the another topic, i.e., the optimal surrender strategies for the EIA investors. Considering a geometric Brownian motion model and infinite horizon, Moore and Young [15] discusses the problem for an investor who may surrender the product and invest directly in the index or banking account, and seeks to maximize the expected utility of wealth at time of death. By including time-dependent contract features, such as the minimum annual growth and the hazard rate, Moore [13] extends the work of Moore and Young [15]. Cheung and Yang [1] studies this problem in a discrete-time model with regime switching. For more discussions of guarantees embedded in insurance products and modeling and pricing issues, we refer to the monograph by Hardy [6].

In this paper, we consider a finite horizon model. As the maturity of EIAs is very long in general, it is more realistic to model the appreciation rate of the equity-linked index by a stochastic process. Similar to Yuen and Yang [20] and Cheung and Yang [1], we capture this feature by considering a regime switching model. In contrast to those papers, we consider a continuous-time model and the Markov chain is not observable. This is more reasonable, since usually the investor can only observable the price of the index directly but not the economic states.

\footnotetext{
"School of Finance and Statistics, East China Normal University, Shanghai, 200241, China. E-mail: jiaqinwei@ gmail.com

${ }^{\dagger}$ School of Finance and Statistics, East China Normal University, Shanghai, 200241, China; School of Mathematics and System Sciences, Shandong University, Jinan, 250100, China. E-mail: rmwang@ @stat.ecnu.edu.cn

Corresponding author. Department of Statistics and Actuarial Science, The University of Hong Kong, Pokfulam Road, Hong Kong, China. E-mail: hlyang@hkusua.hku.hk
} 
The problem considered in this paper is an optimal stopping problem with partial information. There are many papers on this topic in mathematical finance. Under the geometric Brownian motion model with regime switching, Rishel [18] considers a stock holder who can only observable the price of the stock and wants to determine a selling time to maximize logarithmic utility in an infinite time horizon. The optimal stopping time given in Rishel [18] is that the first time the conditional probability process (see Section 2) falls below a threshold whenever such threshold exists; or never stop it if the threshold does not exist. In terms of mathematics, we are considering the same problem as that in Rishel [18], but with a finite time horizon. As we all know, when the horizon is finite, the optimal stopping problem is analytically more difficult, and the optimal stopping time is the first time when the conditional probability process falls below the value of a time-dependent boundary.

In this paper we give a representation of the value function and a integral equation satisfied by the boundary. In the Bayesian case which is a special case of our model, we obtain analytical results for the value function and the boundary. For more details on the optimal stopping under Bayesian model, we refer the reader to Décamps et al. [2], Klein [10] and Ekström and Lu [3].

This paper is organized as follows. In Section 2, we present our model and a preliminary analysis of the optimal stopping problem. In Section 3, we study the optimal stopping problem. We show that the value function is the solution of a free boundary problem and the boundary satisfies a integral equation. In Section 4, we consider the Bayesian case and obtain analytical results.

\section{The Model}

Let us consider the probability space $(\Omega, \mathcal{F}, \mathrm{P})$ and a finite time horizon $T>0$ which is the maturity time of the EIA. Let $J_{t}$ be a Markov chain with two states $\left\{\mathbf{e}_{1}, \mathbf{e}_{2}\right\}$, where $\mathbf{e}_{i}(i=1,2)$ are column vectors with unity in the $i$-th position and zero elsewhere. The state of the chain represent hidden states of an economy. The intensity matrix of $J_{t}$ is given by

$$
\mathbf{Q}=\left(\begin{array}{cc}
-q_{1} & q_{1} \\
q_{2} & -q_{2}
\end{array}\right)
$$

where $q_{1}, q_{2}>0$. We assume that $J_{t}$ is unobservable.

Let $\boldsymbol{\mu}=\left(\mu_{1}, \mu_{2}\right)$, where $\mu_{i}$ is the appreciation rate of the index if the economy is in state $i$, for $i=1,2$. Without loss of generality, we assume that $\mu_{1}>\mu_{2}$. Let $\sigma>0$ denote the constant volatility of the index. The dynamic of the index is given by

$$
\mathrm{d} S_{t}=\mu_{t} S_{t} \mathrm{~d} t+\sigma S_{t} \mathrm{~d} \tilde{W}_{t},
$$

where $\tilde{W}_{t}$ is a standard Brownian motion on $(\Omega, \mathcal{F}, \mathrm{P})$, and $\mu_{t}=\left\langle\mu, J_{t}\right\rangle$.

We assume that the investor can only observe $S_{t}$. Denote by $\mathbb{F}=\left\{\mathcal{F}_{t}^{S}\right\}_{0 \leq t \leq T}$ the filtration generated by $S_{t}$. Now we can define the conditional probability process

$$
X_{t}:=\mathrm{P}\left(J_{t}=\mathbf{e}_{1} \mid \mathcal{F}_{t}^{S}\right), \quad t \geq 0
$$

with initial value $X_{0}=x_{0} \in(0,1)$. Then we have (see, Lipster and Shiryaev [12] and Elliott [4], Chapter $18)$,

$$
\mathrm{d} X_{t}=\left[-q_{1} X_{t}+q_{2}\left(1-X_{t}\right)\right] \mathrm{d} t+\omega\left(1-X_{t}\right) X_{t} \mathrm{~d} W_{t}, \quad t \geq 0,
$$

where $\omega=\left(\mu_{1}-\mu_{2}\right) / \sigma$, and $W_{t}$ is the innovation process which is given by

$$
\mathrm{d} W_{t}=\mathrm{d} \tilde{W}_{t}+\frac{\mu_{t}-\mu_{1} X_{t}-\mu_{2}\left(1-X_{t}\right)}{\sigma} \mathrm{d} t .
$$


It can be shown that $\left(W_{t}, \mathbb{F}\right)$ is a standard P-Brownian motion. The dynamics of the index can be rewritten as

$$
\mathrm{d} S_{t}=\left[\mu_{2}+\left(\mu_{1}-\mu_{2}\right) X_{t}\right] S_{t} \mathrm{~d} t+\sigma S_{t} \mathrm{~d} W_{t} .
$$

Similar to Cheung and Yang [1], for simplicity, the effect of mortality and other product features, like the various embedded guarantees, will be ignored. Without loss of generality, we assume the participation rate is one. Then the evolution of the EIA fund value is governed by

$$
\mathrm{d} Y_{t}=\left[\mu_{2}+\left(\mu_{1}-\mu_{2}\right) X_{t}\right] Y_{t} \mathrm{~d} t+\sigma Y_{t} \mathrm{~d} W_{t}
$$

with $Y_{0}=y_{0}>0$ which is the initial deposit.

At the surrender time $\tau$, the investor receives $Y_{\tau}$. The objective of the investor is to maximize the expected logarithmic utility of the surrender value over all $\mathbb{F}$-stopping time which is bounded by $T$. Then the value function is given by

$$
V_{0}=\sup _{0 \leq \tau \leq T} \mathrm{E}\left[\ln Y_{\tau}\right]
$$

Applying Itô's formula to $\ln Y_{t}$, using (2.2), we have

$$
\ln Y_{t}=\ln Y_{0}+\int_{0}^{t}\left(\mu_{2}+\left(\mu_{1}-\mu_{2}\right) X_{s}-\frac{1}{2} \sigma^{2}\right) \mathrm{d} s+\sigma W_{t}
$$

Hence, the original problem (2.3) is equivalent to the problem

$$
V=\sup _{0 \leq \tau \leq T} \mathrm{E}\left[\int_{0}^{\tau}\left(\mu_{2}+\left(\mu_{1}-\mu_{2}\right) X_{s}-\frac{1}{2} \sigma^{2}\right) \mathrm{d} s\right] .
$$

Note that for all $t \in[0, T]$, the solution of (2.1) satisfies

$$
\mathrm{P}\left(0<X_{t}<1\right)=1,
$$

see, e.g. Rishel [18]. If $\sigma^{2} \geq 2 \mu_{1}$, then the expectation in (2.4) is nonpositive and it is optimal to surrender immediately. If $\sigma^{2} \leq 2 \mu_{2}$, then the expectation in (2.4) is nonnegative and it is optimal to hold the EIA to the maturity. To rule out both of these cases, in the following we assume that $2 \mu_{2}<\sigma^{2}<2 \mu_{1}$.

In order to solve problem (2.4), we are going to study the optimal stopping problem

$$
V(t, x)=\sup _{0 \leq \tau \leq T-t} \mathrm{E}\left[\int_{0}^{\tau}\left(\mu_{2}+\left(\mu_{1}-\mu_{2}\right) X_{s}^{x}-\frac{1}{2} \sigma^{2}\right) \mathrm{d} s\right], \quad(t, x) \in[0, T] \times(0,1),
$$

where $X^{x}$ is a solution of (2.1) with initial value $X_{0}=x$. Note that $V=V\left(0, x_{0}\right)$.

\section{Analysis of the Optimal Stopping Problem}

In this section, we are going to study the optimal stopping problem (2.5). The techniques used in this section are similar to that in Jacka [7], Karatzas and Shreve [9], Section 2.7.

Proposition 3.1. The function $V(\cdot, \cdot):[0, T] \times(0,1) \rightarrow(0, \infty)$ is continuous.

Proof. It follows from (2.1) that

$$
\mathrm{E}\left[X_{t}\right]=X_{0}+\mathrm{E}\left[\int_{0}^{t}\left(q_{2}-\left(q_{1}+q_{2}\right) X_{s}\right) \mathrm{d} s\right] .
$$

Let $0<x_{1} \leq x_{2}<1$. By the comparison result (see Karatzas and Shreve [8], Chapter 5), we know that

$$
\mathrm{P}\left(X_{s}^{x_{1}} \leq X_{s}^{x_{2}}, \forall s \in[0, T-t]\right)=1 .
$$


From the general theory of optimal stopping (see, e.g., Karatzas and Shreve [9], Appendix D and Peskir and Shiryaev [17]), there is an optimal stopping time $\tau$ for $V\left(t, x_{2}\right)$ in the sense that

$$
V\left(t, x_{2}\right)=\mathrm{E}\left[\int_{0}^{\tau}\left(\mu_{2}+\left(\mu_{1}-\mu_{2}\right) X_{s}^{x_{2}}-\frac{1}{2} \sigma^{2}\right) \mathrm{d} s\right] .
$$

Then we have

$$
\begin{aligned}
0 & \leq V\left(t, x_{2}\right)-V\left(t, x_{1}\right) \\
& \leq \mathrm{E}\left[\int_{0}^{\tau}\left(\mu_{2}+\left(\mu_{1}-\mu_{2}\right) X_{s}^{x_{2}}-\frac{1}{2} \sigma^{2}\right) \mathrm{d} s\right]-\mathrm{E}\left[\int_{0}^{\tau}\left(\mu_{2}+\left(\mu_{1}-\mu_{2}\right) X_{s}^{x_{1}}-\frac{1}{2} \sigma^{2}\right) \mathrm{d} s\right] \\
& =\mathrm{E}\left[\int_{0}^{\tau}\left(\mu_{1}-\mu_{2}\right)\left(X_{s}^{x_{2}}-X_{s}^{x_{1}}\right) \mathrm{d} s\right] .
\end{aligned}
$$

Thus, using (3.1), we have

$$
\begin{aligned}
0 & \leq V\left(t, x_{2}\right)-V\left(t, x_{1}\right) \\
& \leq-\frac{\mu_{1}-\mu_{2}}{q_{1}+q_{2}}\left(\mathrm{E}\left[X_{\tau}^{x_{2}}-X_{\tau}^{x_{1}}\right]-\left(x_{2}-x_{1}\right)\right) \\
& \leq \frac{\mu_{1}-\mu_{2}}{q_{1}+q_{2}}\left(x_{2}-x_{1}\right),
\end{aligned}
$$

which implies that $V$ is Lipschitz continuous (uniformly) in $x$.

Let $0 \leq t_{1} \leq t_{2} \leq T$ and $x \in(0,1)$. Let $\tau_{1}$ be an optimal stopping time for $V\left(t_{1}, x\right)$, and define $\tau_{2}=\tau_{1} \wedge\left(T-t_{2}\right)$. It is easy to see that $0 \leq \tau_{1}-\tau_{2} \leq t_{2}-t_{1}$. We then have

$$
\begin{aligned}
0 & \leq V\left(t_{1}, x\right)-V\left(t_{2}, x\right) \\
& \leq \mathrm{E}\left[\int_{0}^{\tau_{1}}\left(\mu_{2}+\left(\mu_{1}-\mu_{2}\right) X_{s}^{x}-\frac{1}{2} \sigma^{2}\right) \mathrm{d} s\right]-\mathrm{E}\left[\int_{0}^{\tau_{2}}\left(\mu_{2}+\left(\mu_{1}-\mu_{2}\right) X_{s}^{x}-\frac{1}{2} \sigma^{2}\right) \mathrm{d} s\right] \\
& =\mathrm{E}\left[\int_{\tau_{2}}^{\tau_{1}}\left(\mu_{1}-\mu_{2}\right) X_{s}^{x} \mathrm{~d} s+\left(\mu_{2}-\frac{1}{2} \sigma^{2}\right)\left(\tau_{1}-\tau_{2}\right)\right] \\
& \leq\left(\mu_{1}-\mu_{2}\right)\left(t_{2}-t_{1}\right)+\left(\mu_{2}-\frac{1}{2} \sigma^{2}\right)\left(t_{2}-t_{1}\right) \\
& =\left(\mu_{1}-\frac{1}{2} \sigma^{2}\right)\left(t_{2}-t_{1}\right) .
\end{aligned}
$$

Recall that we are considering the case with $2 \mu_{1}>\sigma^{2}$. So, we have proved that $V$ is Lipschitz continuous (uniformly) in $t$. This finishes the proof.

Now, define the continuation region $C$ to be

$$
C=\{(t, x) \in[0, T) \times(0,1): V(t, x)>0\},
$$

and the stopping region $\mathcal{D}$ to be

$$
\mathcal{D}=\{(t, x) \in[0, T) \times(0,1): V(t, x)=0\} .
$$

According to the general theory for optimal stopping problems, the stopping time

$$
\tau_{\mathcal{D}}=\inf \left\{0 \leq s \leq T-t:\left(t+s, X_{s}^{x}\right) \in \mathcal{D}\right\}
$$

is an optimal stopping time in (2.5). Therefore, in the following, we are going to determine the stopping region $\mathcal{D}$. 
Remark 3.1. It is easy to see that

$$
\begin{aligned}
V(t, x) & \geq \mathrm{E}\left[\int_{0}^{T-t}\left(\mu_{2}+\left(\mu_{1}-\mu_{2}\right) X_{s}^{x}-\frac{1}{2} \sigma^{2}\right) \mathrm{d} s\right] \\
& =\left(\mu_{2}-\frac{1}{2} \sigma^{2}\right)(T-t)+\left(\mu_{1}-\mu_{2}\right)(T-t) \mathrm{E}\left[X_{s}^{x}\right],
\end{aligned}
$$

where $0 \leq s \leq T-t$. Then, from (3.1), we have

$$
V(t, x) \geq\left(\mu_{2}-\frac{1}{2} \sigma^{2}\right)(T-t)+\left(\mu_{1}-\mu_{2}\right)(T-t)\left[x-q_{1}(T-t)\right] .
$$

Therefore, if $x>b^{*}+q_{1}(T-t)$ where $b^{*}=\frac{\sigma^{2}-2 \mu_{2}}{2\left(\mu_{1}-\mu_{2}\right)}$, we have $V(t, x)>0$.

Proposition 3.2. There exists a non-decreasing and right continuous function $b(\cdot):[0, T) \rightarrow\left[0, b^{*}\right]$, such that

$$
C=\{(t, x) \in[0, T) \times(0,1): x>b(t)\} .
$$

Proof. For some fixed $t \in[0, T)$, and $0<x_{1} \leq x_{2}<1$, suppose that $\left(t, x_{1}\right) \in C$. To prove the existence of $b(t)$, it is sufficient to show that $\left(t, x_{2}\right) \in C$. Indeed, since $V(t, x)$ is increasing in $x$, it is easy to show that $\left(t, x_{2}\right) \in C$.

Moreover, for $0 \leq t_{1} \leq t_{2}<T$ and $x \in(0,1)$, we have $V\left(t_{1}, x\right) \geq V\left(t_{2}, x\right)$. If $x>b\left(t_{2}\right)$ which implies $V\left(t_{2}, x\right)>0$, we have $V\left(t_{1}, x\right) \geq V\left(t_{2}, x\right)>0$ and $x>b\left(t_{1}\right)$. Thus, we have proved that $b(t)$ is increasing.

Since $V(t, x)$ is continuous, $C$ is open. Thus $\mathcal{D}$ is closed. If we take a sequence $\left\{t_{n}\right\} \searrow t$, we see that $\forall n \geq 1,\left(t_{n}, b\left(t_{n}\right)\right) \in \mathcal{D}$. Thus $b(t+) \leq b(t)$. By the increasing nature of $b$, we conclude that $b$ is right continuous.

From Remark 3.1, we know that $b(t) \leq b^{*}+q_{1}(T-t)$. Since $b(t)$ is increasing, for all $t \in[0, T)$, we have $b(t) \leq b^{*}$.

Theorem 3.1. The value function $V(t, x)$ is the unique solution of the initial-boundary value problem

$$
\begin{cases}\mathcal{L} v(t, x)=0, & \text { if } x>b(t), \\ v(t, x)=0, & \text { if } x \leq b(t), \text { or } t=T,\end{cases}
$$

where

$$
\mathcal{L} v=\mu_{1} x+\mu_{2}(1-x)-\frac{1}{2} \sigma^{2}+v_{t}+\left[-q_{1} x+q_{2}(1-x)\right] v_{x}+\frac{1}{2} \omega^{2}(1-x)^{2} x^{2} v_{x x} .
$$

In particular, the partial derivatives $V_{t}, V_{x}$ and $V_{x x}$ exist and are continuous in $C$.

Proof. Obviously, $V$ satisfies the second relation of (3.4). In order to verify the first relation for $V$, we follow the proof of Theorem 2.7.7 of Karatzas and Shreve [9]. Let us take a point $(t, x) \in C$ and a rectangle $\mathcal{R}=\left(t_{1}, t_{2}\right) \times\left(x_{1}, x_{2}\right)$ with $(t, x) \in \mathcal{R} \subset C$. Denoted by $\partial_{0} \mathcal{R}=\partial R \backslash\left[\left\{t_{1}\right\} \times\left(x_{1}, x_{2}\right)\right]$ the parabolic boundary of $\mathcal{R}$. Now consider the initial value problem

$$
\begin{cases}\mathcal{L} v(t, x)=0, & \text { if }(t, x) \in \mathcal{R}, \\ v(t, x)=V(t, x), & \text { if }(t, x) \in \partial_{0} \mathcal{R} .\end{cases}
$$

By Theorem 3.6 of Friedman [5], Chapter 6, there exists an unique solution $v$ to this problem, with $v_{t}, v_{x}$ and $v_{x x}$ continuous. If we show that $V(t, x)=v(t, x)$ in $\mathcal{R}$, then the first relation of (3.4) follows.

Let $\left(t_{0}, x_{0}\right) \in \mathcal{R}$ be given, define the stochastic process

$$
N_{t}^{t_{0}}:=v\left(t_{0}+t, X_{t}^{x_{0}}\right)+\int_{0}^{t}\left(\mu_{2}+\left(\mu_{1}-\mu_{2}\right) X_{s}^{x_{0}}-\frac{1}{2} \sigma^{2}\right) \mathrm{d} s, \quad t \in\left[0, T-t_{0}\right],
$$


and define the stopping time

$$
\tau:=\inf \left\{s \in\left[0, T-t_{0}\right]:\left(t_{0}+s, X_{s}^{x_{0}}\right) \in \partial_{0} \mathcal{R}\right\} .
$$

Applying the Itô's formula yields that $N_{t \wedge \tau}$ is a bounded martingale. Thus

$$
v\left(t_{0}, x_{0}\right)=N_{0}^{t_{0}}=\mathrm{E}\left[N_{\tau}^{t_{0}}\right]=\mathrm{E}\left[M_{\tau}^{t_{0}}\right]
$$

where

$$
M_{t}^{t_{0}}:=V\left(t_{0}+t, X_{t}^{x_{0}}\right)+\int_{0}^{t}\left(\mu_{2}+\left(\mu_{1}-\mu_{2}\right) X_{s}^{x_{0}}-\frac{1}{2} \sigma^{2}\right) \mathrm{d} s .
$$

Define the stopping time

$$
\tau_{t_{0}}:=\inf \left\{s \in\left[0, T-t_{0}\right]: X_{s}^{x_{0}} \leq b\left(t_{0}+s\right)\right\} .
$$

From the general theory of optimal stopping, we know that $M_{t}^{t_{0}}$ is martingale in $\left[0, \tau_{t_{0}}\right]$. Since $\left(t_{0}, x_{0}\right) \in$ $\mathcal{C}$, we have $\tau \leq \tau_{t_{0}}$. The optimal sampling theorem yields that

$$
v\left(t_{0}, x_{0}\right)=M_{0}^{t_{0}}=V\left(t_{0}, x_{0}\right) .
$$

Therefore, $v$ and $V$ agree on $\mathcal{R}$, and hence $V_{t}, V_{x}$ and $V_{x x}$ are defined, continuous, and satisfies the first relation of (3.4) at the arbitrary point $(t, x) \in C$.

For uniqueness, let $v$ be a solution of (3.4). Clearly, $v$ is bounded on $\bar{C}$. For any $t_{0} \in[0, T]$ and $x_{0}>b\left(t_{0}\right)$, applying Itô formula yields that $N_{t \wedge \tau_{t_{0}}}^{t_{0}}$ is a martingale. Thus,

$$
\begin{aligned}
v\left(t_{0}, x_{0}\right)=N_{0}^{t_{0}} & =\mathrm{E}\left[N_{\tau_{t_{0}}}^{t_{0}}\right] \\
& =\mathrm{E}\left[\int_{0}^{\tau_{t_{0}}}\left(\mu_{2}+\left(\mu_{1}-\mu_{2}\right) X_{s}^{x_{0}}-\frac{1}{2} \sigma^{2}\right) \mathrm{d} s\right] \\
& =V\left(t_{0}, x_{0}\right),
\end{aligned}
$$

where the last quality follows from that $\tau_{t_{0}}$ is the optimal stopping time for $V\left(t_{0}, x_{0}\right)$.

Proposition 3.3. The function $V$ is $C^{1}$ in $x$.

Proof. It is sufficient to show that $V_{x}(t, x)$ is continuous at $b(t)$. Since $V_{x}(t, b(t)-)=0$, we only need to show $V_{x}(t, b(t)+)=0$. For any $\varepsilon>0$, let

$$
\tau_{\varepsilon}:=\inf \left\{0 \leq s \leq T-t: X_{s}^{b(t)+\varepsilon} \leq b(t+s)\right\} .
$$

From (3.2), we have

$$
\begin{aligned}
0 & \leq V(t, b(t)+\varepsilon)-V(t, b(t)) \\
& \leq \mathrm{E}\left[\int_{0}^{\tau_{\varepsilon}}\left(\mu_{1}-\mu_{2}\right)\left(X_{s}^{b(t)+\varepsilon}-X_{s}^{b(t)}\right) \mathrm{d} s\right] \\
& =\mathrm{E}\left[\tau_{\varepsilon}\left(\mu_{1}-\mu_{2}\right)\left(X_{s_{\varepsilon}}^{b(t)+\varepsilon}-X_{s_{\varepsilon}}^{b(t)}\right)\right]
\end{aligned}
$$

where $s_{\varepsilon} \in\left[0, \tau_{\varepsilon}\right]$. It follows from (3.3) that

$$
0 \leq V(t, b(t)+\varepsilon)-V(t, b(t)) \leq \varepsilon \mathrm{E}\left[\tau_{\varepsilon}\left(\mu_{1}-\mu_{2}\right)\right] .
$$

Since $b(t)$ is increasing, we have

$$
\tau_{\varepsilon} \leq \tau_{\varepsilon}^{*}:=\inf \left\{0 \leq s \leq T-t: X_{s}^{b(t)+\varepsilon} \leq b(t)\right\} .
$$

However, $\tau_{\varepsilon}^{*} \rightarrow 0$, as $\varepsilon \rightarrow 0$. Dividing (3.5) by $\varepsilon$ and letting $\varepsilon \rightarrow 0$, we obtain $V_{x}(t, b(t)+)=0$. 
Proposition 3.4. The boundary $b(t)$ is continuous on $[0, T)$, and $b(T-)=b^{*}$.

Proof. It follows from Proposition 3.2 that $b$ is right continuous and increasing on $[0, T)$. In order to prove the left continuity, define $b(T)=b^{*}$. We shall suppose that $b\left(t_{0}-\right)<b\left(t_{0}\right)$ for some $t_{0} \in(0, T]$, and obtain a contradiction.

Under the assumption $b\left(t_{0}-\right)<b\left(t_{0}\right)$, there exits $\delta>0$ such that $b\left(t_{0}-\right)<b_{1}<b\left(t_{0}\right)$, where

$$
b_{1}=b^{*}-\frac{\delta}{\mu_{1}-\mu_{2}} .
$$

Let $t \in\left[0, t_{0}\right)$ and $x \in\left(b(t), b_{1}\right)$ be given. Noting that $V(t, x)$ is non-increasing with respective to $t$, it follows from Theorem 3.1 that

$$
\begin{aligned}
\frac{1}{2} \omega^{2}\left(1-x^{2}\right) x^{2} V_{x x}(t, x) & \geq-\left[\mu_{1} x+\mu_{2}(1-x)-\frac{1}{2} \sigma^{2}\right]+\left[q_{1} x-q_{2}(1-x)\right] V_{x}(t, x) \\
& \geq \delta-q_{2} V_{x}(t, x) .
\end{aligned}
$$

Since $0<b(t)<x<b_{1}<1$, we have

$$
\begin{aligned}
V_{x x}(t, x) & \geq \frac{2 \delta}{\omega^{2}\left(1-x^{2}\right) x^{2}}-\frac{2 q_{2}}{\omega^{2}\left(1-x^{2}\right) x^{2}} V_{x}(t, x) \\
& \geq \frac{2 \delta}{\omega^{2}}-\frac{2 q_{2}}{\omega^{2}\left(1-b_{1}^{2}\right) b(t)^{2}} V_{x}(t, x) .
\end{aligned}
$$

Therefore for $x_{0} \in\left(b\left(t_{0}-\right), b_{1}\right)$,

$$
\begin{aligned}
V\left(t, x_{0}\right) & =\int_{b(t)}^{x_{0}} \int_{b(t)}^{y} V_{x x}(t, z) \mathrm{d} z \mathrm{~d} y \\
& \geq \int_{b(t)}^{x_{0}} \int_{b(t)}^{y}\left[\frac{2 \delta}{\omega^{2}}-\frac{2 q_{2}}{\omega^{2}\left(1-b_{1}^{2}\right) b(t)^{2}} V_{x}(t, z)\right] \mathrm{d} z \mathrm{~d} y \\
& =\frac{\delta}{\omega^{2}}\left(x_{0}-b(t)\right)^{2}-\frac{2 q_{2}}{\omega^{2}\left(1-b_{1}^{2}\right) b(t)^{2}} \int_{b(t)}^{x_{0}} V(t, y) \mathrm{d} y .
\end{aligned}
$$

Letting $t \nearrow t_{0}$ and using the continuity of $V$, we obtain

$$
\begin{aligned}
V\left(t_{0}, x_{0}\right) & \geq \frac{\delta}{\omega^{2}}\left(x_{0}-b\left(t_{0}-\right)\right)^{2}-\frac{2 q_{2}}{\omega^{2}\left(1-b_{1}^{2}\right) b\left(t_{0}-\right)^{2}} \int_{b\left(t_{0}-\right)}^{x_{0}} V\left(t_{0}, y\right) \mathrm{d} y \\
& =\frac{\delta}{\omega^{2}}\left(x_{0}-b\left(t_{0}-\right)\right)^{2} \\
& >0
\end{aligned}
$$

which implies that $\left(t_{0}, x_{0}\right) \in C$. But $x_{0}<b_{1}<b\left(t_{0}\right)$, and we obtain a contradiction. Therefore $b(t)$ is continuous on $[0, T)$, and $b(T-)=b^{*}$.

The following proposition characterizes the value function $V(t, x)$ and the boundary $b(t)$. For simplicity, we assume $V$ is regular enough to apply the Itô's formula (see Peskir [16] and Ekström and Lu [3]). However, without this assumption, a proof can be given by using similar method as that in Karatzas and Shreve [9], Theorem 2.7.9.

Proposition 3.5. The function $V(t, x)$ admits the representation

$$
V(t, x)=\int_{0}^{T-t} \mathrm{E}\left[\mathbf{1}_{\left\{X_{s}^{x}>b(t+s)\right\}}\left(\left(\mu_{1}-\mu_{2}\right) X_{s}^{x}+\mu_{2}-\frac{1}{2} \sigma^{2}\right)\right] \mathrm{d} s,
$$

where the boundary $b(t)$ satisfies

$$
0=\int_{0}^{T-t} \mathrm{E}\left[\mathbf{1}_{\left\{X_{s}^{b(t)}>b(t+s)\right\}}\left(\left(\mu_{1}-\mu_{2}\right) X_{s}^{b(t)}+\mu_{2}-\frac{1}{2} \sigma^{2}\right)\right] \mathrm{d} s .
$$


Proof. Fix a $t \in[0, T]$ and $X_{0}=x \in(0, t)$. Applying Itô's formula to $V\left(t+s, X_{s}^{x}\right)$ yields

$$
\begin{aligned}
& V\left(T, X_{T-t}^{x}\right)-V(t, x) \\
= & \int_{0}^{T-t} V_{t}\left(t+s, X_{s}^{x}\right) \mathrm{d} t+\int_{0}^{T-t}\left[-q_{1} X_{s}^{x}+q_{2}\left(1-X_{s}^{x}\right)\right] V_{x}\left(t+s, X_{s}^{x}\right) \mathrm{d} s \\
& +\int_{0}^{T-t} \omega\left(1-X_{s}^{x}\right) X_{s}^{x} V_{x}\left(t+s, X_{s}^{x}\right) \mathrm{d} W_{s}+\frac{1}{2} \int_{0}^{T-t} \omega^{2}\left(1-X_{s}^{x}\right)^{2}\left(X_{s}^{x}\right)^{2} V_{x x}\left(t+s, X_{s}^{x}\right) \mathrm{d} s .
\end{aligned}
$$

By Theorem 3.1, taking the expected value of the above equation gives

$$
V(t, x)=\int_{0}^{T-t} \mathrm{E}\left[\mathbf{1}_{\left\{X_{s}^{x}>b(t+s)\right\}}\left(\left(\mu_{1}-\mu_{2}\right) X_{s}^{x}+\mu_{2}-\frac{1}{2} \sigma^{2}\right)\right] \mathrm{d} s .
$$

Letting $x=b(t)$ in (3.6) yields (3.7).

Remark 3.2. Using similar techniques to Peskir [16], we also can show that $b(t)$ is the unique solution of (3.7). Also, we can prove that the pair $(V, b)$ is the unique solution to (3.4) (see e.g. Karatzas and Shreve [9], Section 2.7).

\section{The Bayesian Case}

In this section, we study (3.6) and (3.7) in a special case with the intensity matrix $\mathbf{Q}=0$ in the model of previous section. This is the so-called Bayesian case where the unobserved drift process $\mu_{t}$ is simply a random variable $\mu_{t}=\mu$, and (2.1) is rewritten as

$$
\mathrm{d} X_{t}=\omega\left(1-X_{t}\right) X_{t} \mathrm{~d} W_{t}, \quad t \geq 0,
$$

with $X_{0}=x_{0} \in(0,1)$. To calculate (3.6) and (3.7) analytically, let $\phi_{t}=X_{t} /\left(1-X_{t}\right)$. Applying the Itô formula yields that

$$
\mathrm{d} \phi_{t}=\omega^{2} X_{t} \phi_{t} \mathrm{~d} t+\omega \phi_{t} \mathrm{~d} W_{t} .
$$

First, by a similar method as that in Klein [10] and Ekström and Lu [3], we introduce a new probability measure $\mathrm{P}^{*}$. To do this, define a new process $W^{*}$ by

$$
\mathrm{d} W_{t}^{*}=\omega X_{t} \mathrm{~d} t+\mathrm{d} W_{t} .
$$

The new probability measure is defined by

$$
\begin{aligned}
\left.\frac{\mathrm{dP}^{*}}{\mathrm{dP}}\right|_{\mathcal{F}_{T}^{s}} & =\exp \left\{-\frac{1}{2} \int_{0}^{T} \omega^{2} X_{t}^{2} \mathrm{~d} t-\int_{0}^{T} \omega X_{t} \mathrm{~d} W_{t}\right\} \\
& =\exp \left\{\frac{1}{2} \int_{0}^{T} \omega^{2} X_{t}^{2} \mathrm{~d} t-\int_{0}^{T} \omega X_{t} \mathrm{~d} W_{t}^{*}\right\} .
\end{aligned}
$$

By Girsanov's theorem, $W^{*}$ is a $\mathrm{P}^{*}$-Brownian motion, and

$$
\mathrm{d} \phi_{t}=\omega \phi_{t} \mathrm{~d} W_{t}^{*}
$$

under measure $\mathrm{P}^{*}$. Define the likelihood process

$$
\eta_{t}=\exp \left\{-\frac{1}{2} \int_{0}^{T} \omega^{2} X_{t}^{2} \mathrm{~d} t+\int_{0}^{T} \omega X_{t} \mathrm{~d} W_{t}^{*}\right\}
$$

which is a $\mathrm{P}^{*}$-martingale. It can be shown that

$$
\eta_{t}=\frac{1+\phi_{t}}{1+\phi_{0}}
$$


(see Ekström and Lu [3]).

Let $\mathrm{E}^{*}$ be the expectation with respective to $\mathrm{P}^{*}$. Noting that $X_{t}=\phi_{t} /\left(1+\phi_{t}\right)$, and using (4.3), we have

$$
\begin{aligned}
& \mathrm{E}\left[\mathbf{1}_{\left\{X_{s}^{x}>b(t+s)\right\}}\left(\left(\mu_{1}-\mu_{2}\right) X_{s}^{x}+\mu_{2}-\frac{1}{2} \sigma^{2}\right)\right] \\
= & \frac{1}{1+\phi_{0}} \mathrm{E}\left[\left(1+\phi_{0}\right) \mathbf{1}_{\left\{X_{s}^{x}>b(t+s)\right\}}\left(\left(\mu_{1}-\mu_{2}\right) X_{s}^{x}+\mu_{2}-\frac{1}{2} \sigma^{2}\right)\right] \\
= & \frac{1}{1+\phi_{0}} \mathrm{E}^{*}\left[\eta_{T}\left(1+\phi_{0}\right) \mathbf{1}_{\left\{\frac{\phi_{s}}{1+\phi_{s}}>b(t+s)\right\}}\left(\left(\mu_{1}-\mu_{2}\right) \frac{\phi_{s}}{1+\phi_{s}}+\mu_{2}-\frac{1}{2} \sigma^{2}\right)\right] \\
= & \frac{1}{1+\phi_{0}} \mathrm{E}^{*}\left[\eta_{s}\left(1+\phi_{0}\right) \mathbf{1}_{\left\{\frac{\phi_{s}}{1+\phi_{s}}>b(t+s)\right\}}\left(\left(\mu_{1}-\mu_{2}\right) \frac{\phi_{s}}{1+\phi_{s}}+\mu_{2}-\frac{1}{2} \sigma^{2}\right)\right] \\
= & \frac{1}{1+\phi_{0}} \mathrm{E}^{*}\left[\left(1+\phi_{s}\right) \mathbf{1}_{\left\{\frac{\phi_{s}}{1+\phi_{s}}>b(t+s)\right\}}\left(\left(\mu_{1}-\mu_{2}\right) \frac{\phi_{s}}{1+\phi_{s}}+\mu_{2}-\frac{1}{2} \sigma^{2}\right)\right] \\
= & \frac{\mu_{1}-\frac{1}{2} \sigma^{2}}{1+\phi_{0}} \mathrm{E}^{*}\left[\mathbf{1}_{\left\{\phi_{s}>\frac{b(t+s)}{1-b(t+s)}\right)} \phi_{s}\right]+\frac{\mu_{2}-\frac{1}{2} \sigma^{2}}{1+\phi_{0}} \mathrm{E}^{*}\left[\mathbf{1}_{\left\{\phi_{s}>\frac{b(t+s)}{1-b(t+s)}\right\}}\right],
\end{aligned}
$$

where $\phi_{0}=x /(1-x)$. From (4.2), we know that

$$
\phi_{t}=\phi_{0} e^{-\frac{1}{2} \omega^{2} t+\omega W_{t}^{*}}
$$

Thus we have

$$
\mathrm{E}^{*}\left[\mathbf{1}_{\left\{\phi_{s}>\frac{b(t+s)}{1-b(t+s)}\right\}} \phi_{s}\right]=\phi_{0} N\left(d_{1}(t, s)\right), \quad \text { and } \quad \mathrm{E}^{*}\left[\mathbf{1}_{\left\{\phi_{s}>\frac{b(t+s)}{1-b(t+s)}\right\}}\right]=N\left(d_{2}(t, s)\right)
$$

where $N(\cdot)$ is the cumulative distribution function of the standard normal distribution, and

$$
\begin{aligned}
& d_{1}(t, s)=\frac{\ln \left[\phi_{0}(1-b(t+s))\right]-\ln b(t+s)}{\omega \sqrt{s}}+\frac{\omega \sqrt{s}}{2}, \\
& d_{2}(t, s)=d_{1}(t, s)-\omega \sqrt{s} .
\end{aligned}
$$

Consequently, we have

$$
V(t, x)=\int_{0}^{T-t}\left[\frac{\phi_{0}}{1+\phi_{0}}\left(\mu_{1}-\frac{1}{2} \sigma^{2}\right) N\left(d_{1}(t, s)\right)+\frac{1}{1+\phi_{0}}\left(\mu_{2}-\frac{1}{2} \sigma^{2}\right) N\left(d_{2}(t, s)\right)\right] \mathrm{d} s,
$$

where $\phi_{0}=x /(1-x)$. Setting $x=b(t)$ gives

$$
0=\int_{0}^{T-t}\left[b(t)\left(\mu_{1}-\frac{1}{2} \sigma^{2}\right) N\left(d_{1}^{*}(t, s)\right)+(1-b(t))\left(\mu_{2}-\frac{1}{2} \sigma^{2}\right) N\left(d_{2}^{*}(t, s)\right)\right] \mathrm{d} s,
$$

where

$$
\begin{aligned}
& d_{1}^{*}(t, s)=\frac{\ln [b(t)(1-b(t+s))]-\ln [(1-b(t)) b(t+s)]}{\omega \sqrt{s}}+\frac{\omega \sqrt{s}}{2}, \\
& d_{2}^{*}(t, s)=d_{1}^{*}(t, s)-\omega \sqrt{s} .
\end{aligned}
$$

Thus in the Bayesian case we can rewrite the results of Proposition 3.5 in a analytical form giving by (4.4) and (4.5). 


\section{Acknowledgments}

We would like to thank the referee for valuable comments and suggestions. This work was supported by National Natural Science Foundation of China (10971068), Doctoral Program Foundation of the Ministry of Education of China (20110076110004), Program for New Century Excellent Talents in University(NCET-09-0356) and the Fundamental Research Funds for the Central Universities, the PHD Program Scholarship Fund of ECNU (No. 2010050), the Ministry Reward for Excellent Doctors in Academics (No. MXRZZ2010006), the Research Grants Council of the Hong Kong Special Administrative Region, China (project No. HKU 706611P).

\section{References}

[1] K. Cheung and H. Yang. Optimal stopping behavior of equity-linked investment products with regime switching. Insurance: Mathematics and Economics, 37:599-614, 2005.

[2] J.-P. Décamps, T. Mariotti, and S. Villeneuve. Investment timing under incomplete information. Math. Oper. Res., 30(2):472-500, 2005.

[3] E. Ekström and B. Lu. Optimal selling of an asset under incomplete information. Submitted, 2010.

[4] R.J. Elliott. Stochastic Calculus and Applications. Springer, Berlin, 1982.

[5] A. Friedman. Stochastic Differential Equations and Applications I. Academic Press, New York, 1975.

[6] M. Hardy. Investment Guarantees: The New Science of Modeling and Risk Management for EquityLinked Insurance. John Wiley, New York, 2003.

[7] S. Jacka. Optimal stopping and the american put. Mathematical Finance, 1(2):1-14, 1991.

[8] I. Karatzas and S. Shreve. Brownian motion and stochastic calculus. Springer-Verlag, New York, second edition, 1991.

[9] I. Karatzas and S. Shreve. Methods of mathematical finance. Springer-Verlag, New York, 1998.

[10] M. Klein. Comment on "investment timing under incomplete information". Math. Oper. Res., 34 (1):249-254, 2009.

[11] X. Lin and K. Tan. Valuation of equity-indexed annuities under stochastic interest rates. North American Actuarial Journal, 7(4):72-91, 2003.

[12] R.S. Lipster and A.N. Shiryaev. Statistics of Random Processes I General Theory. Springer-Verlag, New York, second edition, 2001.

[13] K. Moore. Optimal surrender strategies for equity-indexed annuity investors. Insurance: Mathematics and Economics, 44:1-18, 2009.

[14] K. Moore and V. Young. Pricing equity-indexed pure endowments via the principle of equivalent utility. Insurance: Mathematics and Economics, 33:497-516, 2003.

[15] K. Moore and V. Young. Optimal design of a perpetual equity-indexed annuity. North American Actuarial Journal, 9(1):57-72, 2005.

[16] G. Peskir. On the american option problem. Math. Finance, 15:169-181, 2005. 
[17] G. Peskir and A. Shiryaev. Optimal Stopping and Free-Boundary Problems. Birkhäuser, Basel, 2006.

[18] R. Rishel. Whether to sell or hold a stock. Communications in Information and Systems, 6:193$202,2001$.

[19] S. Tiong. Valuing equity-indexed annuities. North American Actuarial Journal, 4(4):149-163, 2000.

[20] F.L. Yuen and H. Yang. pricing asian options and equity-indexed annuities with regime switching by the trinomial tree method. The North American Actuarial Journal, 14(2):256-277, 2010. 